\title{
A Key Major Guideline for Engineering Bioactive Multicomponent Nanofunctionalization for Biomedicine and Other Applications: Fundamental Models Confirmed by Both Direct and Indirect Evidence
}

\author{
Roya Dastjerdi, ${ }^{1}$ Andreas Scherrieble, ${ }^{2}$ Shiva Bahrizadeh, ${ }^{1}$ \\ Fatemeh Avareh Sadrabadi, ${ }^{1}$ and Laleh Hedayat ${ }^{3}$ \\ ${ }^{1}$ Textile Engineering Department, Yazd University, Yazd, Iran \\ ${ }^{2}$ German Institutes of Textile and Fiber Research Denkendorf, Koerschtalstrasse 26, 73770 Denkendorf, Germany \\ ${ }^{3}$ Microbiological Laboratory, Boomazma Co., Yazd Science and Technology Park, Yazd, Iran \\ Correspondence should be addressed to Roya Dastjerdi; nanobiotex@yahoo.com
}

Received 3 August 2017; Accepted 4 October 2017; Published 29 November 2017

Academic Editor: Jinsong Ren

Copyright (C) 2017 Roya Dastjerdi et al. This is an open access article distributed under the Creative Commons Attribution License, which permits unrestricted use, distribution, and reproduction in any medium, provided the original work is properly cited.

\begin{abstract}
This paper deals with the engineering multicomponent nanofunctionalization process considering fundamental physicochemical features of nanostructures such as surface energy, chemical bonds, and electrostatic interactions. It is pursued by modeling the surface nanopatterning and evaluating the proposed technique and the models. To this end, the effects of surface modifications of nanoclay on surface interactions, orientations, and final features of $\mathrm{TiO}_{2} / \mathrm{Mt}$ nanocolloidal textiles functionalization have been investigated. Various properties of cross-linkable polysiloxanes (XPs) treated samples as well as untreated samples with XPs have been compared to one another. The complete series of samples have been examined in terms of bioactivity and some physical properties, given to provide indirect evidence on the surface nanopatterning. The results disclosed a key role of the selected factors on the final features of treated surfaces. The effects have been thoroughly explained and modeled according to the fundamental physicochemical features. The developed models and associated hypotheses interestingly demonstrated a full agreement with all measured properties and were appreciably confirmed by FESEM evidence (direct evidence). Accordingly, a guideline has been developed to facilitate engineering and optimizing the pre-, main, and post-multicomponent nanofunctionalization procedures in terms of fundamental features of nanostructures and substrates for biomedical applications and other approaches.
\end{abstract}

\section{Introduction}

A novel approach, after raveling the excellent properties of nanostructures [1-25], is the use of multicomponent formulation of nanostructures [26-33] to achieve outstanding features. Designing a good engineered process to apply different kinds of nanostructures, accompanied with each other, can facilitate achieving the desirable properties. Surface features of biomaterial like roughness, surface electrical charges, wetting behavior, and so forth can determine the cell response and biocompatibility as required in different biomedical approaches $[34,35]$. The desired modification in these surface features can be designed by a well-engineered multicomponent nanofunctionalization. However, the critical challenge is finding the key factors and approaches for predicting, engineering, and modeling the multicomponent nanofunctionalization which can be used as a guideline for future designing. Among nanostructures with different shapes and geometries, nanoparticles and nanolayers are the most used species. Among the nanoparticles (NPs), $\mathrm{TiO}_{2}$ [36-41], and among the nanolayers (NLs), clay [42-44], are the most reported nanostructures in biomedical approaches as well as other applications. They are the safest nanostructures enjoying remarkable properties and a wide range of applications [45]. Moreover, a certain bioinspired nanoroughness and/or a synergistic effect can be accomplished by 
TABLE 1: Introducing different samples.

\begin{tabular}{lcccc}
\hline Sample code & $\begin{array}{c}\text { Pretreatment } \\
\text { (Mercerizing) }\end{array}$ & Natural NLs & Nanolayer functionalization \\
Organomodified NLs & $\begin{array}{c}\text { Nanoparticle functionalization } \\
\text { TiO }\end{array}$ & NPs \\
C & $X$ & $X$ & $X$ & $X$ \\
M.C & $\checkmark$ & $X$ & $X$ & $X$ \\
NL & $X$ & $\checkmark$ & $\checkmark$ & $X$ \\
ONL & $X$ & $X$ & $X$ & $X$ \\
M.NL & $\checkmark$ & $\checkmark$ & $\checkmark$ & $X$ \\
M.ONL & $\checkmark$ & $X$ & $X$ & $\checkmark$ \\
NP & $X$ & $X$ & $X$ & $\checkmark$ \\
M.NP & $\checkmark$ & $X$ & $X$ & $\checkmark$ \\
NL/NP & $X$ & $\checkmark$ & $\checkmark$ \\
ONL/NP & $X$ & $X$ & $\checkmark$ \\
M.NL/NP & $\checkmark$ & $\checkmark$ & $\checkmark$ & $\checkmark$ \\
M.ONL/NP & $\checkmark$ & $X$ & $\checkmark$ & $\checkmark$ \\
\hline
\end{tabular}

an appropriate multicomponent nanopatterning on surface of biomedical membranes or devises.

Given the importance of the aforementioned approaches in the case of multicomponent nanofunctionalization, here, the $\mathrm{TiO}_{2}$-containing nanolayered silicate, montmorillonite (Mt), as an isotropic/anisotropic mixed nanocolloid, has been used to achieve multiple-size nanoroughness, as well as multifunctional properties via ultrasound-assistant exhaustion process on textiles, followed by the polysiloxane posttreatment. Above all, a key major guideline on engineering multicomponent nanofunctionalization has been proposed considering fundamental physicochemical features of nanostructures such as surface energy, chemical bonds, and electrostatic interactions. The fundamental models were confirmed by both direct and indirect evidence. By achieving this goal, a guideline has been developed to facilitate engineering the nanofunctionalization procedures of biomaterials and other supplies.

\section{Experimental Section}

2.1. Materials. Natural montmorillonite, Cloisite ${ }^{\circledR} \mathrm{Na}^{+}$, and organomodified montmorillonite (Cloisite 30B: sodium montmorillonite modified by methyl, tallow, bis-2-hydroxyethyl, quaternary ammonium chloride), supplied by Southern Clay Company, were used to prepare the intercalated nanolayer colloidal solutions. $\mathrm{TiO}_{2}$ (P25 nano-titanium dioxide) was kindly provided by Evonik (Degussa) Corporation. $\mathrm{TiO}_{2}$ P25 contains $80 \mathrm{wt} \%$ anatase and $20 \mathrm{wt} \%$ rutile structures and has 25-30 nm particle size [46]. Polysiloxane CT 208 E emulsion was kindly provided by Wacker Finish. CT $208 \mathrm{E}$ is a water-based polysiloxane emulsion, a commercially available product, by Wacker Finish (Germany). It contains $66 \%$ solid content containing $60 \%$ active substance content of aminofunctional polysiloxane, as stated by supplier.

2.2. Methods. At first, colloidal solutions of $0.1 \mathrm{wt} \%$ natural or organomodified clay nanolayers (NLs or ONLs) have been prepared using magnetic stirrer and ultrasonic waves. Then, keeping the dispersing powers, the $\mathrm{TiO}_{2}$ nanoparticles (NPs,
$0.1 \mathrm{wt} \%$ ) have been gradually added to the clay colloidal solutions. The cotton fabrics have been treated via exhaustion process under ultrasonic waves for $45 \mathrm{~min}$. Some samples have been also designed applying only NLs, ONLs, or NPs, to compare the effect of each component. The produced samples have been coded by their treatment components as NL, ONL, NP, NL/NP, and ONL/NP (Table 1). All the production steps have been performed at ambient temperature and atmosphere. The posttreatment by XPs was performed as follows. Each sample was immersed in $2 \mathrm{wt} \%$ polysiloxane solution for $5 \mathrm{~s}$ at ambient temperature and atmosphere and squeezed by pad to $100 \%$ wet pick-up. Then, the padded samples were dried at $100^{\circ} \mathrm{C}$. The process has been performed on mercerized and unmercerized cotton fabrics with the same structures to investigate the pretreatment effect. The mercerized samples were indicated by adding "M." prior to their code.

\subsection{Characterizations}

2.3.1. Hydrophobicity. The hydrophobicity of samples was studied, according to AATCC Standard Test Method 79, by measuring the time required for water droplet to be completely spread on the fabric surfaces. To this end, water was dropped from $1 \mathrm{~cm}$ on the fabric surface by a small syringe. The time of the complete absorption of $10 \mu \mathrm{l}$ water droplets on the fabric surfaces (in $27^{\circ} \mathrm{C}$ and $30 \%$ relative humidity) was measured for 10 replicates after which the average value was reported. Contact angle (CA) of a $10 \mu \mathrm{l}$ distilled water droplet dropped from a distance of $1 \mathrm{~cm}$ was measured 5 seconds after placing on the fabric surface using a self-developed goniometer apparatus equipped with a high resolution camera and suitable lens. The volume of the droplets was exactly controlled using a special capillary connected to an Atom Syringe Pump S-1235, Japan. The average of contact angles of two sides of 6 drops, determined by using MB-Ruler software, was calculated and reported as CA of each sample [47].

2.3.2. Scanning Electron Microscopy. Field emission scanning electron microscope (FESEM) micrographs were obtained 
using a Vega/Tescan, Mira 3-XMU field emission scanning electron microscope with both low and high magnifications. The samples were coated by a thin layer of gold, sputtered on the samples with a thickness of about $30 \mathrm{~nm}$, before FESEM analysis.

2.3.3. Stiffness. The Shirley stiffness test was used as a criterion for stiffness and the bending length was reported for test specimens cut in $25 \mathrm{~mm}$ width and $200 \mathrm{~mm}$ length parallel to the warp. This experiment was performed for all samples with 6 replicates and the average values were reported.

2.3.4. Crease Recovery Angle. The wrinkle recovery angle in the warp and weft fabric directions was measured according to BS 3086: 1972 test method by the Shirley Crease Recovery tester, with 4 replicates, and the average values were reported.

2.3.5. UV Protection Properties. Evaluating the color changes of wool fabrics dyed by a highly UV sensitive dye $(0.5 \mathrm{wt} \%$ Methylene Blue) which were covered by different modified and unmodified samples under UV irradiation was considered as a criterion for the comparison of the UV protection properties of samples [48]. UV irradiations were provided by a Philips Cleo UV lamp HPA 400 S located $25 \mathrm{~cm}$ above the covered samples for $18 \mathrm{~h}$. After UV irradiation, the color changes were studied based on reflectance data using an Xrite spectrophotometer according to the formula reported in our previous paper [48].

2.3.6. Photocatalytic Bioactivity. Bioactivity of the functionalized samples was investigated on the five-time-washed samples via methods exactly reported in our previous papers [48] but with some modifications as follows. The photoirradiation was conducted by a conventional fluorescent lamp (20 W Sinar ${ }^{\circledR}$ Merbabu, Indonesia) located $60 \mathrm{~cm}$ above the samples for 18 hours. $R(\%)$ is the percentage of reduction of bacteria as compared to the untreated sample under the same test conditions, referring to the antibacterial efficiency. The washing procedure on samples was performed using a Rotawash Model M228B Launderometer according to AATCC test method 61(2A)-1996 to 5-cycle washing.

\section{Results and Discussions}

3.1. Hydrophobicity and Stain Repellency. In recent years, developing a bioinspired nanoroughness to achieve the demanded hydrophobicity or hydrophilicity is increasingly investigated $[49,50]$. Wetting behavior of biomedical devices is one of the most important criteria indicating their performance for different applications [51-53]. For instance, an ultra-hydrophilic feature is vital for haemostatic and clotting membranes [54]. Superhydrophobic medical devices can be designed for many other cardiovascular applications such as pericardial substitutes [52], artificial vessels, and stents. Consequently, the appropriate feature must be engineered for each application utilizing innovative techniques.

Wettability of samples treated with nanostructures and XPs can be considered as a criterion indicating the formation of a complete layer of XPs on the nanostructures, promising

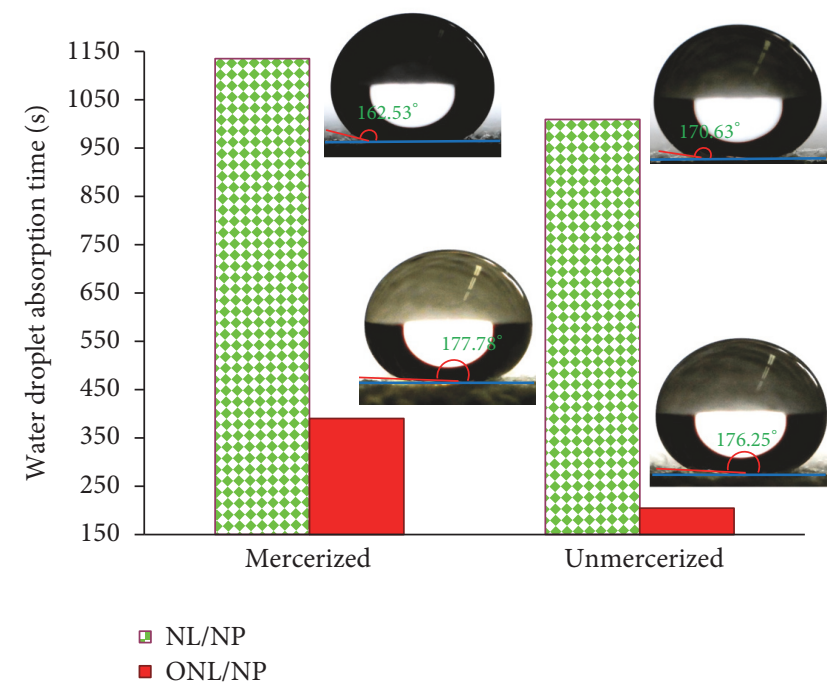

FIGURE 1: The effect of pretreatment (mercerizing) and nanolayer modification on hydrophobicity of XPs-treated samples.

ideal fastness as well as multifunctional properties [48]. In fact, the maximum hydrophobicity can be accomplished by forming a complete layer of XPs. The incomplete layer leads to a reduced hydrophobicity. On the other hand, extra XPs, more than the amount required to form one layer, will reduce the hydrophobicity due to inverse orientation of XPs hydrophilic groups on the first layer. On this basis, this measurement is the most important feature to provide certain information required to determine the appropriate chemical ratios of nanostructures and XPs. Consequently, in a predesigned experiment, the proper XPs ratio has been determined for the nanoparticle-containing intercalated nanolayers produced in this research. According to the unreported results, the best XPs concentration, which reached $2 \mathrm{wt} \%$, was derived and used in this research. The average value of water droplet absorption time for XPs-treated nanofunctionalized samples has been reported in Table 2. The water droplet absorption time for unmodified cotton fabrics was less than one second. Water droplets were also fast spread on all nanofunctionalized samples without XPs treatment. The cross-linkable polysiloxane increased water droplet absorption times on all nanofunctionalized samples (Table 2). The hydrophobic properties achieved by colloidal solution of $\mathrm{TiO}_{2}$ containing nanolayers were more than those achieved by colloidal solution of pure nanolayers without NPs. This result was recorded for both kinds of nanolayers. Generally, creating hydrophobic nanoroughness can increase the hydrophobic properties of surfaces. As mentioned above, XPs coverage with a suitable concentration can provide the hydrophobic feature on the nanofunctionalized samples. Fine nanoroughness can provide more and finer air pocket and increase hydrophobicity [55]. The nanoroughness created by $\mathrm{TiO}_{2}$ nanopatterns on nanolayer was more effective than nanoroughness created by pure nanolayers. The effects of nanoclay types on hydrophobicity of nano- $\mathrm{TiO}_{2} /$ clay functionalized samples have been compared with each other as shown in Figure 1. Unexpectedly, the hydrophobicity created 
TABLE 2: Wetting properties of XPs-treated nanofunctionalized samples.

\begin{tabular}{lcr}
\hline Sample code & Water droplet absorption time $(\mathrm{s})$ & Water droplet contact angle $\left(^{\circ}\right)$ \\
\hline NL & 205 & $149.8 \pm 1.2$ \\
ONL & 118 & $149.8 \pm 1.8$ \\
M.NL & 134 & $150.2 \pm 1.5$ \\
M.ONL & 197 & $148.1 \pm 2.1$ \\
NP & 251 & $148.6 \pm 1.3$ \\
M.NP & 2068 & $>150 \pm 2.5$ \\
NL/NP & 1009 & $>150 \pm 2.5$ \\
ONL/NP & 204 & $>150 \pm 2.5$ \\
M.NL/NP & 1135 & $>150 \pm 2.4$ \\
M.ONL/NP & 390 & $>150 \pm 2.5$ \\
\hline
\end{tabular}

by $\mathrm{TiO}_{2}$ NPs containing natural montmorillonite was significantly more than that of $\mathrm{TiO}_{2}$ containing organomodified montmorillonite. To explain this phenomenon, we should thoroughly consider the whole process. In this research, $\mathrm{TiO}_{2} /$ clay nanocolloidal solutions have been prepared by gradually adding $\mathrm{TiO}_{2}$ NPs to clay nanocolloidal solutions under ultrasound. Since the difference of surface energy levels between natural montmorillonite and $\mathrm{TiO}_{2} \mathrm{NPs}$ is less than that of organomodified montmorillonite and $\mathrm{TiO}_{2} \mathrm{NPs}$, the affinity of nanoparticles to be absorbed on the natural montmorillonite is more than that of the organomodified layers in the colloidal solution. This would be mainly intensified by electrostatic interactions between natural montmorillonite and $\mathrm{TiO}_{2}$ NPs due to the negative surface charge of $\mathrm{TiO}_{2}$ nanoparticles above pH 5.6 (here the solvent was distilled water with $\mathrm{pH}$ about 7) and the positive charge of $\mathrm{Na}^{+}$ions on natural montmorillonite, Cloisite $\mathrm{Na}^{+}$. Consequently, the colloidal solution of natural montmorillonite and $\mathrm{TiO}_{2} \mathrm{NPs}$ can create an intensified nanoroughness on the functionalized fabrics (NL/NP) as shown schematically in Figure 2. Creating this intensified, finer, and more regular nanoroughness can enhance hydrophobicity following the XPs treatment, while nanoparticles and nanolayers are mostly separated in the colloidal solution of organomodified montmorillonite and $\mathrm{TiO}_{2}$ NPs (ONL/NP). Moreover, because of the enhanced affinity of organomodified layers and cotton fiber surfaces, the separated layers are attracted to lie on the fabric surfaces as a result of the hydrophobic-hydrophobic interactions among the organic groups on layers and fiber surfaces, as well as potentiality to form hydrogen bonds between their $\mathrm{OH}$ groups. Therefore, their cooperation to create nanoroughness reduced as compared to natural montmorillonite. These hypotheses have been interestingly confirmed by microscopic evidence. The FESEM micrographs have been demonstrated in Figures 3 and 4. The water droplet absorption time for XPs-treated mercerized samples was more than that of unmercerized samples. This pointed out the effect of this conventional pretreatment on increasing the reactivity intensifying of adsorption of nanostructures during the nanofunctionalization process. As is well known, mercerizing causes some structural changes in cotton fibers. The reduction of crystallinity as a result of mercerizing leads to the increase of the amorphous regions which have

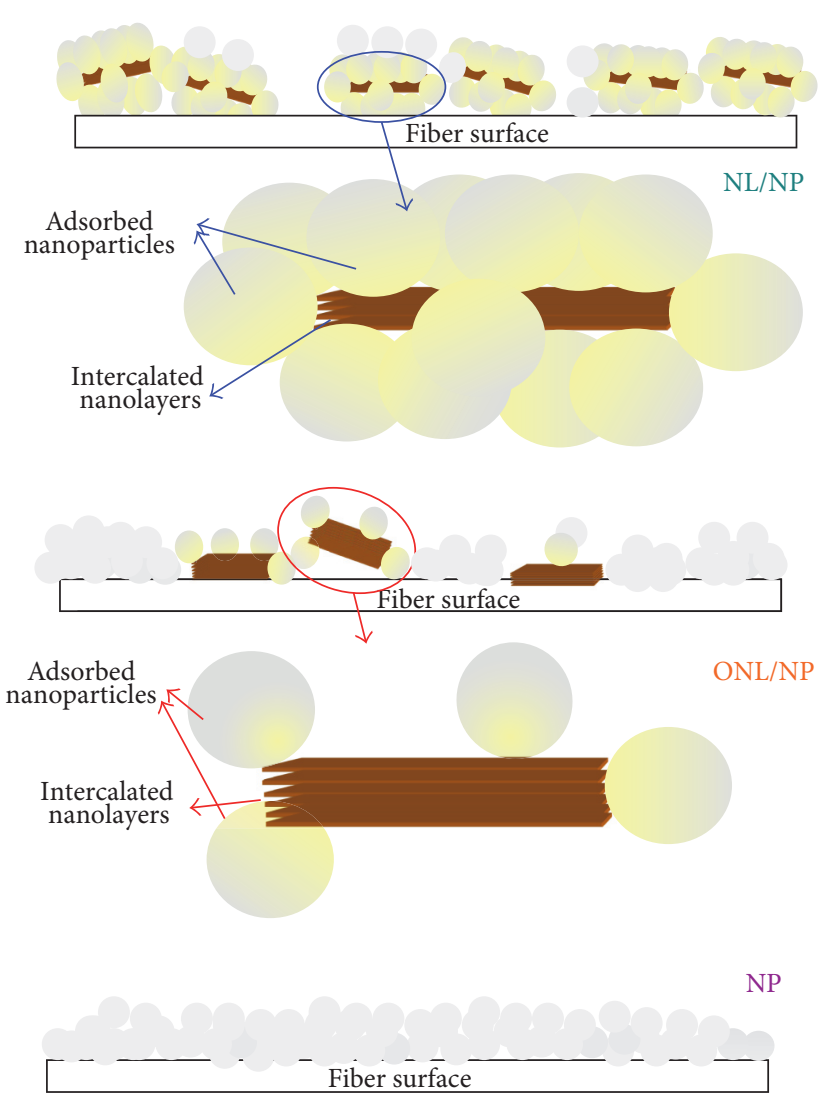

FIGURE 2: Schematic model for surface orientation of nanostructures on nanolayer/nanoparticle (NL/NP), organomodified nanolayer/nanoparticle (ONL/NP), and nanoparticle (NP) functionalized samples.

a good potentiality for the adsorption of nanostructures. Increasing fibrous porosity, moisture regains, fabric hygroscopicity, absorbency, smoother morphology, and so forth caused by this pretreatment "mercerizing" [56, 57] can direct the better adsorption of nanostructures on mercerized fabrics. The maximum hydrophobicity has been obtained for XPs-treated mercerized sample functionalized with only $\mathrm{TiO}_{2}$ NPs. Unexpectedly, the enhanced hydrophobicity was reduced by adding nanolayers to nanoparticles. This can be explained by reducing the created nanoroughness due to 


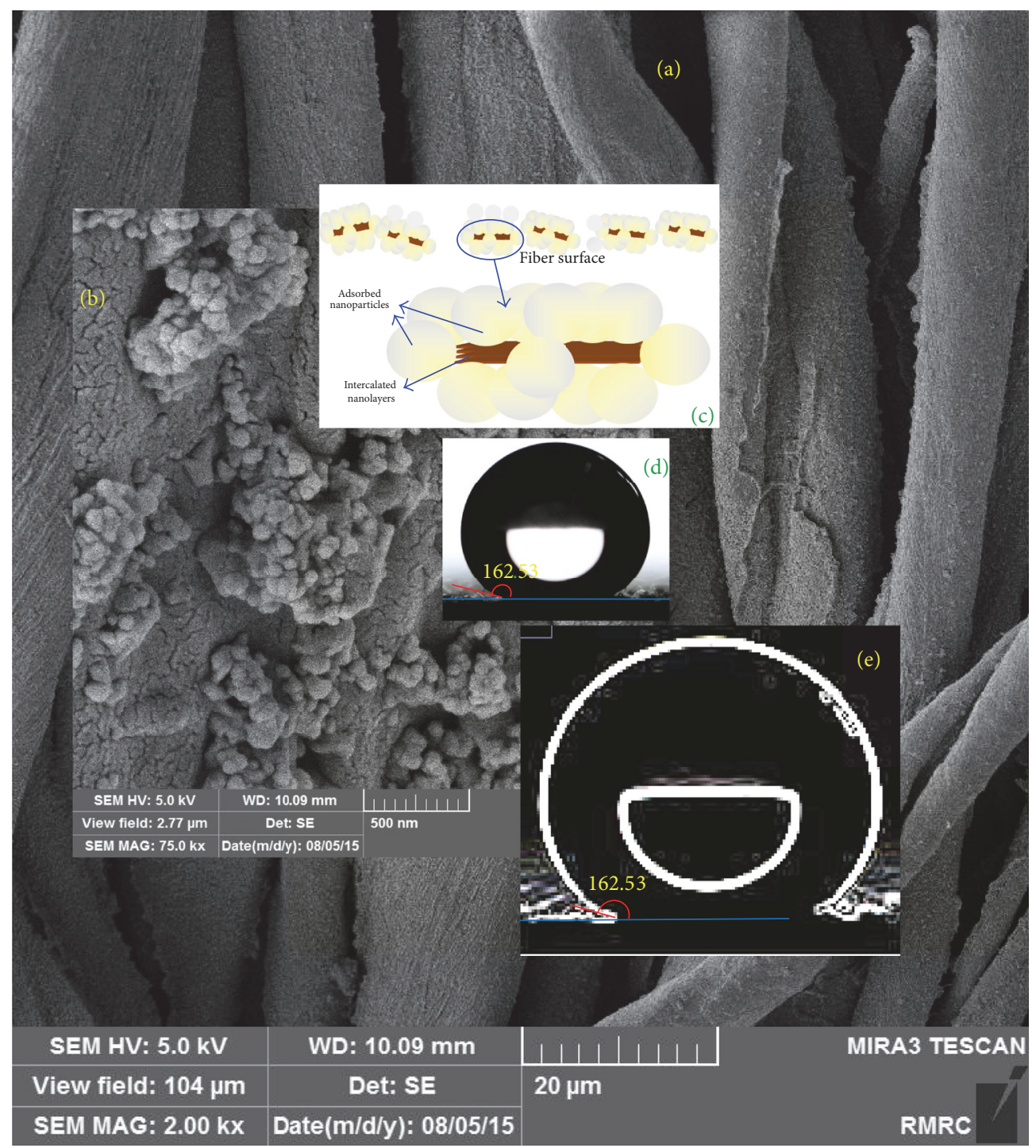

Figure 3: ((a) and (b)) FESEM micrographs of fiber surfaces functionalized by nanocolloidal natural montmorillonite and TiO 2 (NL/NP), with low and high magnification, respectively, (c) schematic model proposed according to the fundamental physic-chemical phenomena for this sample (NL/NP) in comparison with the microscopic outcomes, (d) water droplet image and contact angle $\left({ }^{\circ}\right)$ on this sample, and $(\mathrm{e})$ image "(d)" processed by "the find edge option" of ImageJ software to clarify of the droplet boundaries for precise evaluation of contact angle.

covering some parts of nanoparticle roughness by nanolayers. Moreover, the created roughness in the absence of layers is finer and more regular (see Figure 5), providing smaller air pockets on the interface of fabric and droplets which require a higher level of energy to be overcome [55]. However, all XPs-treated nanofunctionalized samples demonstrate significant increases of absorption times as compared to both untreated mercerized and unmercerized cotton. This enhanced hydrophobicity along with a high contact angle (about $150^{\circ}$, see Table 2) provided a superhydrophobic feature on cotton fabrics (Figure 3 ). The achieved hydrophobicity satisfied the perfect stain repellency. XPs-treated samples can quickly repel and remove the stain droplets before they can be adsorbed, while the stain droplets were completely absorbed on the untreated samples. It should be pointed out that achieving such high contact angle on the textiles substrates is absolutely more difficult than other substrate like glass, plastic sheets or films, steel or other metallic devices or surfaces, and so forth. Numerous research works have also reported achieving a superhydrophobicity using fluorinated compounds and/or with a smaller droplet [58-62]. A recently published paper which has been also considered as a cutting edged publication [63] has yet used organic solvents to apply a fluorine-free treatment to textiles. Moreover, none of its droplet images [63] shows a contact angle bigger than our achievements, even with a smaller droplet [63]. We are reporting a water-based fluorine-free superhydrophobic treatment on cotton fabrics in this paper. The droplet images recorded in our research point to the achievement of a unique contact angle which has not been reported so far either on textiles or on other substrates. For example, the droplet on Figure 4(d) and specially its sharp-edge version (Figure 4(e)), 


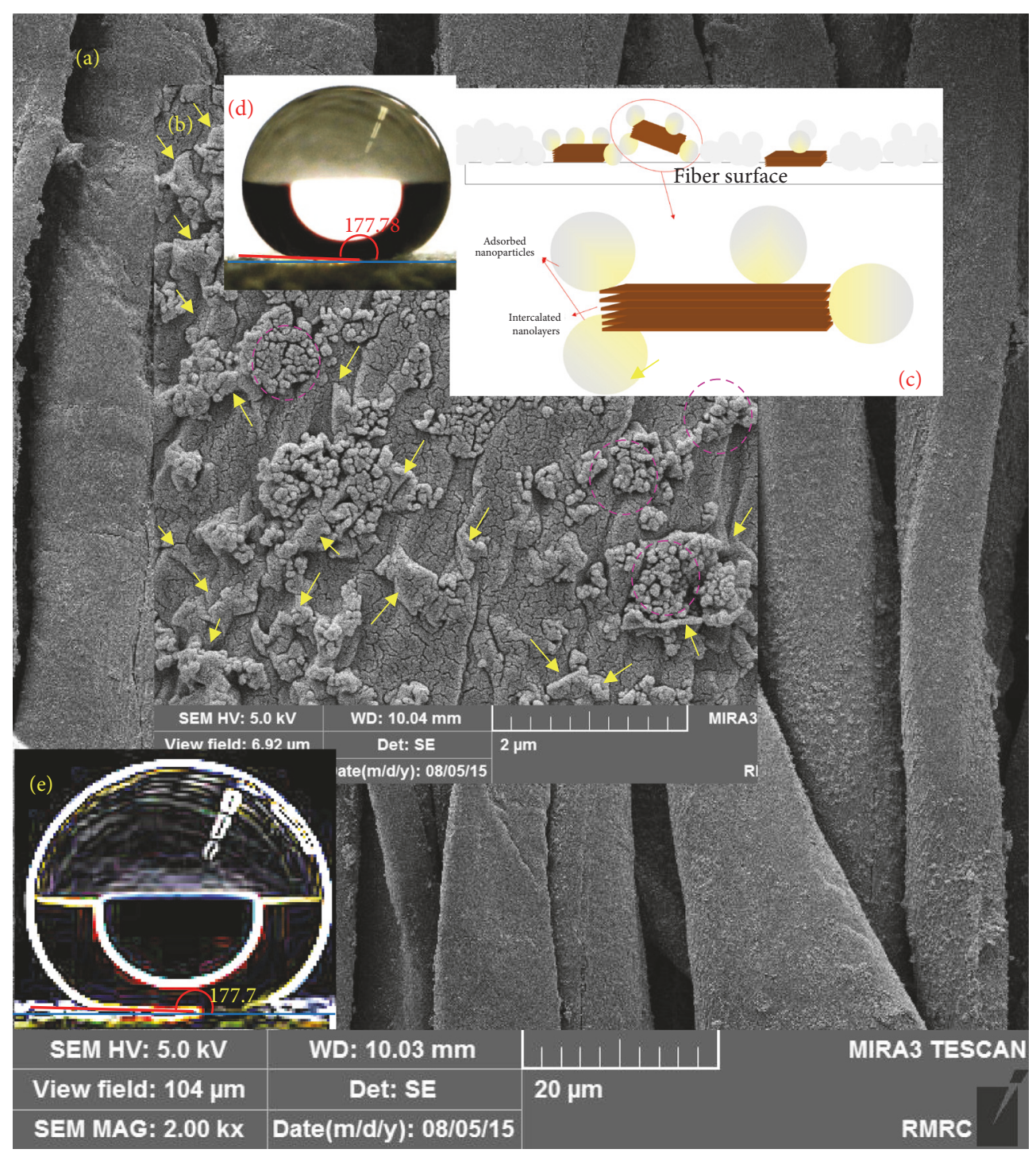

FIGURE 4: ((a) and (b)) FESEM micrographs of fiber surfaces functionalized by nanocolloidal organomodified montmorillonite and $\mathrm{TiO}_{2}$ (ONL/NP), with low and high magnification, respectively; arrows point out separated nanolayers and circles indicate nanoparticles, (c) schematic model proposed according to the fundamental physic-chemical phenomena for this sample (ONL/NP) in comparison with the microscopic outcomes, (d) water droplet image and contact angle $\left(^{\circ}\right)$ on this sample, and (e) image "(d)" processed by "the find edge option" of ImageJ software to clarify the droplet boundaries for precise evaluation of contact angle.

processed by "the find edge option" of ImageJ software to clarify of the droplet boundaries and more precise evaluation, show achieving a contact angle of about $177^{\circ}$. However, we have relinquished this evidence and prudently reported more than $150^{\circ}$ for the cases which were more than $150^{\circ}$ (Table 2), while they (CAs) were notably more for all of the droplet images (see also Figures 1 and 3). Therefore, this treatment can be suggested for developing blood-repellent hospital textiles [64], as well as hygienic home and industrial textiles. The superhydrophobic samples have also a remarkable potentiality for antiadhesive biomedical applications like pericardial substitutes. The achievements in this paper meet the sharpest cutting edged scientific subjects [65].

3.2. Field Emission Scanning Electron Microscopy (FESEM). Field emission scanning electron microscopy (FESEM) was used to study the surface orientation of nanostructures on nanocolloidal functionalized samples. Figures 3(a) and 3(b) show FESEM micrographs of fiber surfaces functionalized by nanocolloidal natural montmorillonite and $\mathrm{TiO}_{2}$. Similarly, Figures 4(a) and 4(b) demonstrate the evidence of organomodified montmorillonite and $\mathrm{TiO}_{2}$. Although cotton fibers naturally pose an uneven morphology, the micrographs with low magnifications, $\mathrm{x}=2000$, shown as background (Figures 3(a) and 4(a)), appreciably corroborated forming an even coating of nanostructures with a good dispersibility on each sample. Figures 3(a) and 4(a) are also implying formation of a thin even coverage of nanomaterials around the fibers surfaces without blocking the fabric holes. In fact, since the size of fabric holes are considerably bigger than the thickness of the formed thin coverage, the fabrics air permeability is not negatively affected by such even treatment 


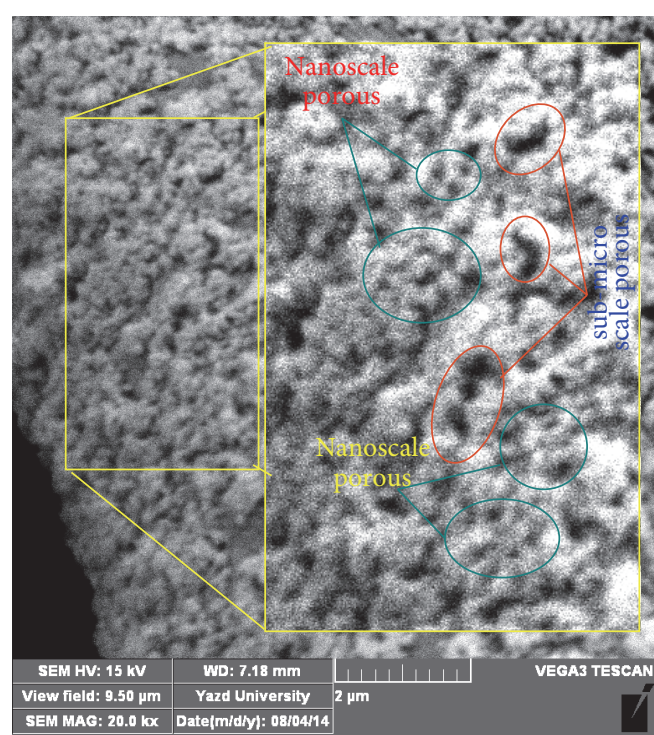

FIGURE 5: SEM micrographs of fiber surfaces functionalized by nanocolloidal $\mathrm{TiO}_{2}$ creating multiscale nano/submicroporous interfaces.

as the previous report [48] also confirms this issue. The appropriate magnifications have been also provided to show the surface orientation of nanostructures for each sample. The given evidence by FESEM surprisingly confirmed all developed models (Figures 2, 3(c), and 4(c)) and associated theories raised based on the wetting properties. As thoroughly discussed in Section 3.1, these models and theories have been yielded according to the results along with considering fundamental features of interfaces, consisting of the differences of surface energy levels between nanostructures, surface charges, and surface interactions of the nanolayers and nanoparticles, as well as their interactions and orientations on textile surfaces. Wonderfully, all achievements are also in complete agreement with all other characteristics discussed in the following sections.

3.3. UV Protection Properties. The results showed that the enhancement of UV protection properties of fabrics functionalized by $\mathrm{TiO}_{2}$ containing organomodified montmorillonite was more than $\mathrm{TiO}_{2}$ containing natural montmorillonite (Figure 6). This implies the intensified cocovering effect of natural nanolayers and nanoparticles due to their electrostatic interactions as thoroughly discussed in Section 3.1, schematically shown in Figure 2, and confirmed by microscopic evidence (Figures 3 and 4).

In addition, as mentioned before, organomodified montmorillonite is attracted to lie on the fabric surfaces due to the hydrophobic-hydrophobic interactions and hydrogenic bonds between the organic groups on layers and fiber surfaces, and in this way, they can provide better support for fiber (see scheme in Figures 2 and 4). This result interestingly confirmed the developed models on the basis of wettability of samples. The enhancement of UV protection properties is also more for $\mathrm{TiO}_{2}$ treated fabrics than that of $\mathrm{TiO}_{2}$ containing nanolayers indicating the cocovering

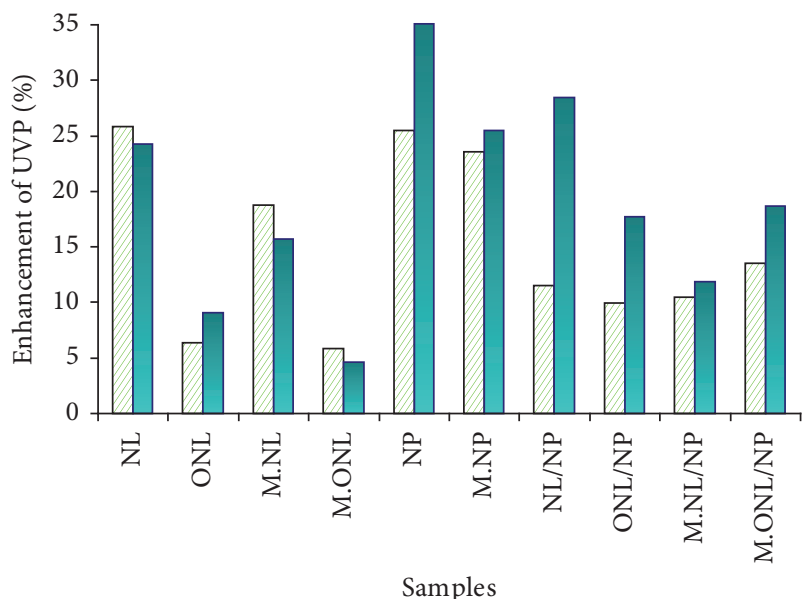

$\square$ Without XPs

口 XPs-treated

FIGURE 6: Enhancement of UV protection properties (\%) as compared to control samples (C and $\mathrm{MC}$ ).

effect of nanolayers and nanoparticles, in agreement with the developed models and results of measuring hydrophobicity. UV protection properties were positively affected by XPs treatment. In fact, XPs treatment can improve the UV protection properties on nanofunctionalized surfaces via covering certain parts of bigger nanoroughness followed by reducing irregular multi-internal reflections [48], increasing the reflection proportion against light transfer [47], inducing light to the $\mathrm{TiO}_{2}$ and clay nanostructures as the UV-absorbent, and increasing the absorption proportion against light transfer.

3.4. Fabric Stiffness. Functionalization of fabric surfaces with inorganic nanostructures can commonly lead to the decrease of fabric softness [48]. Bending length is one of the best indices to decide on the fabric softness. The results showed that the values of bending length of mercerized nanofunctionalized fabrics are generally more than those of unmercerized ones. This confirmed the more reactivity and better adsorption of nanostructures on mercerized cotton, interestingly in agreement with the results and discussions developed in Section 3.1. However, XPs, as a softener, have also compensated for the reduction of fabric softness and reduced the bending length (Table 3 ). Therefore, XPs treatment can promise more comfortable multifunctional textiles via improving softness and handle.

3.5. Anti-Crease Properties. The recovery of wrinkle has been reduced by nanofunctionalization due to steric hindrance of inorganic nanostructures against the movement of polymeric chains (Table 3). However, XPs treatment has compensated for this effect and improved antiwrinkle properties of XPs-treated nanofunctionalized fabrics due to its softening as well as plasticizing effects. Moreover, the formation of network-like layer of polysiloxane on the fabric surfaces [47] can increase their resistance to crease. 
TABLE 3: Bending length and crease recovery angle of different samples.

\begin{tabular}{|c|c|c|c|c|c|c|}
\hline \multirow{2}{*}{ Sample code } & \multicolumn{2}{|c|}{ Bending length $(\mathrm{cm})$} & \multicolumn{2}{|c|}{ Crease recovery angle (warp) $\left(^{\circ}\right)$} & \multicolumn{2}{|c|}{$\begin{array}{c}\text { Crease recovery angle (weft) } \\
\left({ }^{\circ}\right)\end{array}$} \\
\hline & $\begin{array}{l}\text { Before XPs } \\
\text { treatment }\end{array}$ & $\begin{array}{l}\text { After XPs } \\
\text { treatment }\end{array}$ & $\begin{array}{l}\text { Before XPs } \\
\text { treatment }\end{array}$ & $\begin{array}{l}\text { After XPs } \\
\text { treatment }\end{array}$ & $\begin{array}{l}\text { Before XPs } \\
\text { treatment }\end{array}$ & $\begin{array}{l}\text { After XPs } \\
\text { treatment }\end{array}$ \\
\hline $\mathrm{C}$ & 5.4 & 5.7 & 121 & 134 & 122 & 131 \\
\hline M.C & 6.2 & 5.7 & 135 & 131 & 120 & 126 \\
\hline NL & 5.6 & 5.4 & 101 & 122 & 111 & 128 \\
\hline ONL & 5.3 & 5.3 & 107 & 132 & 109 & 126 \\
\hline M.NL & 6.3 & 5.6 & 115 & 126 & 109 & 134 \\
\hline M.ONL & 6.2 & 5.8 & 116 & 118 & 120 & 134 \\
\hline NP & 5.2 & 5.4 & 110 & 128 & 119 & 126 \\
\hline M.NP & 6.4 & 5.7 & 103 & 115 & 105 & 128 \\
\hline NL/NP & 4.2 & 5.2 & 105 & 10 & 107 & 116 \\
\hline ONL/NP & 5.5 & 5.1 & 108 & 130 & 114 & 116 \\
\hline M.NL/NP & 6.3 & 5.8 & 109 & 124 & 113 & 121 \\
\hline M.ONL/NP & 6.1 & 5.6 & 116 & 120 & 103 & 123 \\
\hline
\end{tabular}

3.6. Photo-Bioactivity. The 5-time-washed XPs-treated samples with the best multifunctional properties were chosen for evaluation of their antibacterial activities against Escherichia coli (ATCC 25922). The concentration of the bacteria in the primary inoculums was $2.1 \times 10^{6}$. The number of bacteria recovered from the inoculated untreated sample immediately after inoculation was estimated as $3.7 \times 10^{5} \mathrm{cfu} \cdot \mathrm{ml}^{-1}$. The average number of bacteria recovered from the incubated untreated samples under the photo $\left(81 \times 10^{5} \mathrm{cfu} \cdot \mathrm{ml}^{-1}\right)$ was considered as a reference to calculate the antibacterial activity.

Table 4 reports the antibacterial activity of the tested samples. Sufficient antibacterial efficiency was achieved by the mercerized organomodified montmorillonite and $\mathrm{TiO}_{2}-$ treated sample (M.ONL/NP) as well as the mercerized $\mathrm{TiO}_{2}$ treated sample (M.NP). Although the antibacterial activity of the mercerized sample treated with Cloisite $\mathrm{Na}^{+}$and $\mathrm{TiO}_{2}$ (M.NL/NP) was also satisfying for many applications, it could not compete with the best sample. This can be caused by cocovering of nanostructures as thoroughly discussed before. The results of the designed qualitative test for comparing the antibacterial efficiency of some samples are also exhibited in Figure 7.

The antibacterial activity, along with other practical features of treated samples, as discussed above, makes them an excellent suggestion for hospital textiles [66-68] as well as many hygienic garments and home textiles.

\section{Conclusions}

A guideline to realize the key factors and approaches for predicting, engineering, and modeling the multicomponent nanofunctionalization for biomedical devices or other application has been developed in this research. Moreover, a novel bioactive polysiloxane-shield nanolayer patterned by nanoparticles has been produced and investigated. The key role of the surface properties of applied nanostructures on the
TABLE 4: Bioactivity of washed X-Ps treated samples.

\begin{tabular}{lcc}
\hline Samples & Bacteria number $\times 10^{-5^{*}}$ & $R \%$ \\
\hline MC & 81 & 0.00 \\
M.NP & 1 & 98.77 \\
M.NL/NP & 19.56 & 75.85 \\
M.ONL/NP & 0.44 & 99.46 \\
\hline
\end{tabular}

${ }^{*}$ The average number of $10^{5}$ times diluted bacteria recovered from the inoculated test specimen swatches.

surface patterning and multifunctional properties of treated surfaces has been discussed and modeled according to their surface energy, chemical bonds, and electrostatic interactions. The accomplished models and associated hypotheses have appreciably a full agreement with all the investigated characteristics and have been interestingly confirmed by microscopic evidence. The results and models developed in this paper serve as a major guideline in the following highly interesting topics:

(i) Engineering the pre-, main, and post-multicomponent nanofunctionalization procedures in terms of fundamental features of nanostructures and different surfaces

(ii) Designing novel multicomponent nanocolloids, nanocoatings, and nanocomposites for biological and/ or multifunctional approaches

(iii) Developing discussions and models for multifunctional features of modifications based on multicomponent nanostructures with different geometries including isotropic and anisotropic structures

(iv) Achieving multifunctional properties by polysiloxane-shield nanoparticle-containing intercalated nanolayers on different surfaces

(v) Comparing polysiloxane-shield nanolayer and nanoparticle functionalizations 


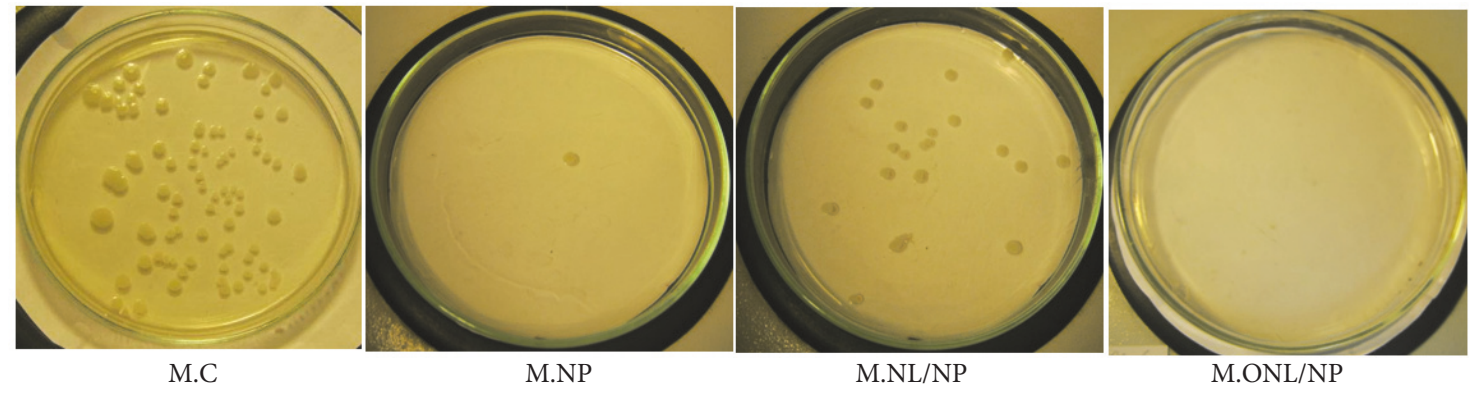

FIGURE 7: Qualitative comparing photo-bioactivity basis of $10^{5}$ times diluted bacteria, recovered from the inoculated test specimen swatches of washed XPs-treated samples.

(vi) Studying the effect of nanolayer surface modifications on the properties of functionalized surfaces

(vii) Comparing the effect of functionalization with nanolayers, nanoparticles, and nanoparticle-containing intercalated nanolayers

(viii) Studying the effect of surface modifications of nanolayers on their patterning by nanoparticles through their interactions, orientations, and so forth

(ix) Investigating the effect of polysiloxane posttreatment in all aforementioned topics via comparing XPstreated samples with samples without XPs treatment

(x) Studying the effect of substrate pretreatment on all aforementioned functionalizations.

\section{Disclosure}

Roya Dastjerdi's other email is nanobiotex.dastjerdi@yazd ac.ir.

\section{Conflicts of Interest}

The authors declare that there are no conflicts of interest regarding the publication of this paper.

\section{Acknowledgments}

The authors would also like to acknowledge the contributions of Evonik (Degussa) Corporation (Germany) and Wacker Finish (Germany) for kindly providing nano- $\mathrm{TiO}_{2}$ and polysiloxane emulsion, respectively.

\section{References}

[1] C. A. Cozzolino, F. Nilsson, M. Iotti, B. Sacchi, A. Piga, and S. Farris, "Exploiting the nano-sized features of microfibrillated cellulose (MFC) for the development of controlled-release packaging," Colloids and Surfaces B: Biointerfaces, vol. 110, pp. 208-216, 2013.

[2] S. Sundarrajan, A. R. Chandrasekaran, and S. Ramakrishna, "An update on nanomaterials-based textiles for protection and decontamination," Journal of the American Ceramic Society, vol. 93, no. 12, pp. 3955-3975, 2010.
[3] K. Hadinoto, A. Sundaresan, and W. S. Cheow, "Lipid-polymer hybrid nanoparticles as a new generation therapeutic delivery platform: A review," European Journal of Pharmaceutics and Biopharmaceutics, vol. 85, no. 3, pp. 427-443, 2013.

[4] C. Stacchi, T. Lombardi, F. Oreglia, A. Alberghini Maltoni, and T. Traini, "Histologic and histomorphometric comparison between sintered nanohydroxyapatite and anorganic bovine xenograft in maxillary sinus grafting: a split-mouth randomized controlled Clinical Trial," BioMed Research International, vol. 2017, Article ID 9489825, 10 pages, 2017.

[5] G. Rajagopal, S. Maruthamuthu, S. Mohanan, and N. Palaniswamy, "Biocidal effects of photocatalytic semiconductor $\mathrm{TiO}_{2}$," Colloids and Surfaces B: Biointerfaces, vol. 51, no. 2, pp. 107-111, 2006.

[6] A. Patsidis, S. N. Georga, C. A. Krontiras, G. C. Psarras, A. Kanapitsas, and C. Tsonos, "Effect of filler size on the thermal properties of ER/BaTiO3 composites," Macromolecular Symposia, vol. 331-332, no. 1, pp. 189-196, 2013.

[7] K. Hadinoto and W. S. Cheow, "Nano-antibiotics in chronic lung infection therapy against Pseudomonas aeruginosa," Colloids and Surfaces B: Biointerfaces, vol. 116, pp. 772-785, 2014.

[8] M. S. Islam and J. H. Yeum, "Electrospun pullulan/poly(vinyl alcohol)/silver hybrid nanofibers: preparation and property characterization for antibacterial activity," Colloids and Surfaces A: Physicochemical and Engineering Aspects, vol. 436, no. 5, pp. 279-286, 2013.

[9] E. I. Salama, A. Abbas, and A. M. Esawi, "Preparation and properties of dual-matrix carbon nanotube-reinforced aluminum composites," Composites Part A: Applied Science and Manufacturing, vol. 99, pp. 84-93, 2017.

[10] J.-L. Chen, F.-L. Jin, and S.-J. Park, "Thermal stability and impact and flexural properties of epoxy resins/epoxidized castor oil/nano-CaCO3 ternary systems," Macromolecular Research, vol. 18, no. 9, pp. 862-867, 2010.

[11] Y. I. Yoon, K. E. Park, S. J. Lee, and W. H. Park, "Fabrication of microfibrous and nano-/microfibrous scaffolds: Melt and hybrid electrospinning and surface modification of poly $(\mathrm{L}-$ lactic acid) with plasticizer," BioMed Research International, vol. 2013, Article ID 309048, 10 pages, 2013.

[12] S. S. Mano, K. Kanehira, S. Sonezaki, and A. Taniguchi, "Effect of polyethylene glycol modification of $\mathrm{TiO} 2$ nanoparticles on cytotoxicity and gene expressions in human cell lines," International Journal of Molecular Sciences, vol. 13, no. 3, pp. 3703-3737, 2012. 
[13] X. Liang, Y. Rangom, C. Y. Kwok, Q. Pang, and L. F. Nazar, "Interwoven MXene Nanosheet/Carbon-Nanotube Composites as Li-S Cathode Hosts," Advanced Materials, vol. 29, no. 3, Article ID 1603040, 2017.

[14] K. Kandori, S. Oda, M. Fukusumi, and Y. Morisada, "Synthesis of positively charged calcium hydroxyapatite nano-crystals and their adsorption behavior of proteins," Colloids and Surfaces B: Biointerfaces, vol. 73, no. 1, pp. 140-145, 2009.

[15] Y. Lu, M. Yu, M. Drechsler, and M. Ballauff, "Ag nanocomposite particles: Preparation, characterization and application," Macromolecular Symposia, vol. 254, pp. 97-102, 2007.

[16] J. M. Fuentes-Alventosa, L. Introzzi, N. Santo, G. Cerri, A. Brundu, and S. Farris, "Self-assembled nanostructured biohybrid coatings by an integrated 'sol-gel/intercalation' approach," RSC Advances, vol. 3, no. 47, pp. 25086-25096, 2013.

[17] M. S. Sataev, S. T. Koshkarbaeva, A. B. Tleuova, S. Perni, S. B. Aidarova, and P. Prokopovich, "Novel process for coating textile materials with silver to prepare antimicrobial fabrics," Colloids and Surfaces A: Physicochemical and Engineering Aspects, vol. 442, pp. 146-151, 2014.

[18] P. Chen, S. Migita, K. Kanehira, S. Sonezaki, and A. Taniguchi, "Development of sensor cells using NF- $\kappa$ B pathway activation for detection of nanoparticle-induced inflammation," Sensors, vol. 11, no. 7, pp. 7219-7230, 2011.

[19] D. Lee, S. Kim, B. Kim, S. Chun, S. Lee, and Q. Wu, "Effect of nano-CaCO3 and Talc on property and weathering performance of PP composites," International Journal of Polymer Science, vol. 2017, Article ID 4512378, 9 pages, 2017.

[20] R. Sridhar, S. Sundarrajan, A. Vanangamudi, G. Singh, T. Matsuura, and S. Ramakrishna, "Green processing mediated novel polyelectrolyte nanofibers and their antimicrobial evaluation," Macromolecular Materials and Engineering, vol. 299, no. 3, pp. 283-289, 2014.

[21] P. Chen, H.-S. Kim, and H.-J. Jin, "Preparation, properties and application of poly amide/carbon nanotube nanocomposites," Macromolecular Research, vol. 17, no. 4, pp. 207-217, 2009.

[22] R. Ravichandran, R. Sridhar, J. R. Venugopal, S. Sundarrajan, S. Mukherjee, and S. Ramakrishna, "Gold nanoparticle loaded hybrid nanofibers for cardiogenic differentiation of stem cells for infarcted myocardium regeneration," Macromolecular Bioscience, vol. 14, no. 4, pp. 515-525, 2014.

[23] Y. Gu, Q. Ran, W. She, and J. Liu, "Modifying cement hydration with NS@PCE core-shell nanoparticles,” Advances in Materials Science and Engineering, vol. 2017, Article ID 3823621, 13 pages, 2017.

[24] W. Li, M. Zhao, C. Ke et al., "Nano polymeric carrier fabrication technologies for advanced antitumor therapy," BioMed Research International, vol. 2013, Article ID 305089, 9 pages, 2013.

[25] D. Zhai, T. Zhang, J. Guo, X. Fang, and J. Wei, "Waterbased ultraviolet curable conductive inkjet ink containing silver nano-colloids for flexible electronics," Colloids and Surfaces A: Physicochemical and Engineering Aspects, vol. 424, pp. 1-9, 2013.

[26] H. Zhang, X. Lv, Y. Li, Y. Wang, and J. Li, "P25-graphene composite as a high performance photocatalyst," ACS Nano, vol. 4, no. 1, pp. 380-386, 2010.

[27] D. Zhou, H. Jia, J. Rana et al., "Local structural changes of nano-crystalline $\mathrm{ZnFe} 2 \mathrm{O} 4$ during lithiation and de-lithiation studied by X-ray absorption spectroscopy," Electrochimica Acta, vol. 246, pp. 699-706, 2017.

[28] J. Safaei-Ghomi, S. H. Nazemzadeh, and H. Shahbazi-Alavi, "Nano-CdZr4(PO4)6 as a reusable and robust catalyst for the synthesis of bis-thiazolidinones by a multicomponent reaction of aldehydes, ethylenediamine and thioglycolic acid," Journal of Sulfur Chemistry, vol. 38, no. 2, pp. 195-205, 2017.

[29] J. Virkutyte, S. R. Al-Abed, E. F. Barth, D. Reible, P. Dunlap, and S. Chattopadhyay, "Catalytic Sorption of (Chloro)Benzene and Naphthalene in Aqueous Solutions by Granular Activated Carbon Supported Bimetallic Iron and Palladium Nanoparticles," ISRN Nanotechnology, vol. 2013, Article ID 525697, 8 pages, 2013.

[30] X. Pan, I. Medina-Ramirez, R. Mernaugh, and J. Liu, "Nanocharacterization and bactericidal performance of silver modified titania photocatalyst," Colloids and Surfaces B: Biointerfaces, vol. 77, no. 1, pp. 82-89, 2010.

[31] M. J. Yi, S.-H. Byun, C.-S. Ha, D.-W. Park, and I. Kim, “Copolymerization of cyclohexene oxide with carbon dioxide over nano-sized multi-metal cyanide catalysts," Solid State Ionics, vol. 172, no. 1-4, pp. 139-144, 2004.

[32] A. S. Gouveia-Neto, A. F. Da Silva, L. A. Bueno, and E. B. Da Costa, "Luminescent features of solgel derived rare-earth multidoped oxyfluoride nano-structured phosphors for white LED application," Journal of Luminescence, vol. 132, no. 2, pp. 299304, 2012.

[33] S. Li, T. Zhu, J. Huang, Q. Guo, G. Chen, and Y. Lai, "Durable antibacterial and UV-protectiveAg/TiO2@fabrics for sustainable biomedical application," International Journal of Nanomedicine, vol. 12, pp. 2593-2606, 2017.

[34] A. Albanese, P. S. Tang, and W. C. W. Chan, "The effect of nanoparticle size, shape, and surface chemistry on biological systems," Annual Review of Biomedical Engineering, vol. 14, pp. 1-16, 2012.

[35] R. I. Shebl, F. Farouk, and H. M. Azzazy, "Effect of Surface charge and hydrophobicity modulation on the antibacterial and antibiofilm potential of magnetic iron nanoparticles," Journal of Nanomaterials, vol. 2017, Article ID 528295, 15 pages, 2017.

[36] K. Kanehira, T. Banzai, C. Ogino, N. Shimizu, Y. Kubota, and S. Sonezaki, "Properties of $\mathrm{TiO}_{2}$-polyacrylic acid dispersions with potential for molecular recognition," Colloids and Surfaces B: Biointerfaces, vol. 64, no. 1, pp. 10-15, 2008.

[37] Y. L. Lam, C. W. Kan, C. W. M. Yuen, and C. H. Au, "Fabric objective measurement of the plasma-treated cotton fabric subjected to wrinkle-resistant finishing with BTCA and $\mathrm{TiO}_{2}$ system," Fibers and Polymers, vol. 12, no. 5, pp. 626-634, 2011.

[38] P. Chen, K. Kanehira, S. Sonezaki, and A. Taniguchi, "Detection of cellular response to titanium dioxide nanoparticle agglomerates by sensor cells using heat shock protein promoter," Biotechnology and Bioengineering, vol. 109, no. 12, pp. 3112-3118, 2012.

[39] Y. L. Lam, C. W. Kan, and C. W. M. Yuen, "Physical and chemical analysis of plasma-treated cotton fabric subjected to wrinkleresistant finishing," Cellulose, vol. 18, no. 2, pp. 493-503, 2011.

[40] J. Virkutyte, S. R. Al-Abed, H. Choi, and C. Bennett-Stamper, "Distinct structural behavior and transport of $\mathrm{TiO}_{2}$ nano- and nanostructured particles in sand," Colloids and Surfaces A: Physicochemical and Engineering Aspects, vol. 443, pp. 188-194, 2014.

[41] M. Lorenzetti, A. Drame, S. Šturm, and S. Novak, “TiO2 (nano) particles extracted from sugar-coated confectionery," Journal of Nanomaterials, vol. 2017, Article ID 6298307, 14 pages, 2017.

[42] S. S. Ray and M. Okamoto, "Polymer/layered silicate nanocomposites: a review from preparation to processing," Progress in Polymer Science, vol. 28, no. 11, pp. 1539-1641, 2003. 
[43] M. Aliabadi, R. Dastjerdi, and K. Kabiri, "HTCC-modified nanoclay for tissue engineering applications: A synergistic cell growth and antibacterial efficiency," BioMed Research International, vol. 2013, Article ID 749240, 7 pages, 2013.

[44] B. Baghaei, S. H. Jafari, H. A. Khonakdar, I. Rezaeian, L. As'Habi, and S. Ahmadian, "Interfacially compatibilized LDPE/ POE blends reinforced with nanoclay: Investigation of morphology, rheology and dynamic mechanical properties," Polymer Bulletin, vol. 62, no. 2, pp. 255-270, 2009.

[45] R. Dastjerdi and M. Montazer, "A review on the application of inorganic nano-structured materials in the modification of textiles: focus on anti-microbial properties," Colloids and Surfaces B: Biointerfaces, vol. 79, no. 1, pp. 5-18, 2010.

[46] S. Mozia and A. W. Morawski, "Integration of photocatalysis with ultrafiltration or membrane distillation for removal of azo dye direct green 99 from water," Journal of Advanced Oxidation Technologies, vol. 12, no. 1, pp. 111-121, 2009.

[47] R. Dastjerdi and S. A. Noorian, "Polysiloxane features on different nanostructure geometries; nano-wires and nanoribbons," Colloids and Surfaces A: Physicochemical and Engineering Aspects, vol. 452, no. 1, pp. 25-31, 2014.

[48] R. Dastjerdi, M. Montazer, and S. Shahsavan, "A novel technique for producing durable multifunctional textiles using nanocomposite coating," Colloids and Surfaces B: Biointerfaces, vol. 81, no. 1, pp. 32-41, 2010.

[49] T. Darmanin and F. Guittard, "Recent advances in the potential applications of bioinspired superhydrophobic materials," Journal of Materials Chemistry A, vol. 2, no. 39, pp. 16319-16359, 2014.

[50] Y. K. Lai, L. X. Lin, F. Pan et al., "Bioinspired patterning with extreme wettability contrast on $\mathrm{TiO}_{2}$ nanotube array surface: a versatile platform for biomedical applications," Small, vol. 9, no. 17, pp. 2945-2953, 2013.

[51] N. Cañas, M. Kamperman, B. Völker, E. Kroner, R. M. McMeeking, and E. Arzt, "Effect of nano- and micro-roughness on adhesion of bioinspired micropatterned surfaces," Acta Biomaterialia, vol. 8, no. 1, pp. 282-288, 2012.

[52] S. Amanpour, H. Ahamadi, H. Mirzadeh, M. A. Mohagheghi, and S. Rabbani, "Long-term evaluation of laser-treated silicone (LTS) membrane as a pericardial substitute: In vivo study," Journal of Long-Term Effects of Medical Implants, vol. 15, no. 4, pp. 347-353, 2005.

[53] A. J. Scardino, H. Zhang, D. J. Cookson, R. N. Lamb, and R. de Nys, "The role of nano-roughness in antifouling," Biofouling, vol. 25, no. 8, pp. 757-767, 2009.

[54] H. Takahashi, T. Miyoshi, and K. Boki, "Study on hydrophilic properties of gelatin as a clinical wound dressing. II. Waterabsorbing property and hemostatic effect of gelatin.", Tokushima Journal of Experimental Medicine, vol. 40, no. 3-4, pp. 169175, 1993.

[55] R. Dastjerdi, M. Montazer, T. Stegmaier, and M. B. Moghadam, "A smart dynamic self-induced orientable multiple size nano-roughness with amphiphilic feature as a stain-repellent hydrophilic surface," Colloids and Surfaces B: Biointerfaces, vol. 91, no. 1, pp. 280-290, 2012.

[56] T. L. Vigo, Textile Processing and Properties: Preparation, Dyeing, Finishing and Performance, Elsevier Publishers, New York, NY, USA, 1994.

[57] R. Freytag and J. J. Donze, "Handbook of Fiber Science and Technology," in Volume 1 Chemical Processing of Fibers and Fabrics: Part A Fundamental and Preparation, M. Lewin and S.
B. Sello, Eds., pp. 93-166, Marcel Dekker, New York, NY, USA, 1983.

[58] H. Teisala, M. Tuominen, and J. Kuusipalo, "Superhydrophobic Coatings on Cellulose-Based Materials: Fabrication, Properties, and Applications," Advanced Materials Interfaces, vol. 1, no. 1, Article ID 1300026, 2014.

[59] V. K. Wimalasiri, H. U. Weerathunga, N. Kottegoda, and V. Karunaratne, "Silica based superhydrophobic nanocoatings for natural rubber surfaces," Journal of Nanomaterials, vol. 2017, Article ID 2102467, 14 pages, 2017.

[60] M. Kiuru and E. Alakoski, "Low sliding angles in hydrophobic and oleophobic coatings prepared with plasma discharge method," Materials Letters, vol. 58, no. 16, pp. 2213-2216, 2004.

[61] F. Li, M. Du, Z. Zheng, Y. Song, and Q. Zheng, "A Facile, Multifunctional, Transparent, and Superhydrophobic Coating Based on a Nanoscale Porous Structure Spontaneously Assembled from Branched Silica Nanoparticles," Advanced Materials Interfaces, vol. 2, no. 13, Article ID 1500201, 2015.

[62] H. Liu, J. Huang, F. Li et al., "Multifunctional superamphiphobic fabrics with asymmetric wettability for one-way fluid transport and templated patterning," Cellulose, vol. 24, no. 2, pp. 1129-1141, 2017.

[63] C. Cao, M. Ge, J. Huang et al., "Robust fluorine-free superhydrophobic PDMS-ormosil@fabrics for highly effective selfcleaning and efficient oil-water separation," Journal of Materials Chemistry A, vol. 4, no. 31, pp. 12179-12187, 2016.

[64] J.-S. Cho and G. Cho, "Effect of a dual function finish containing an antibiotic and a fluorochemical on the antimicrobial properties and blood repellency of surgical gown materials," Textile Research Journal, vol. 67, no. 12, pp. 875-880, 1997.

[65] S. Li, J. Huang, Z. Chen, G. Chen, and Y. Lai, "A review on special wettability textiles: theoretical models, fabrication technologies and multifunctional applications," Journal of Materials Chemistry A, vol. 5, no. 1, pp. 31-55, 2017.

[66] S. Lee, J.-S. Cho, and G. Cho, "Antimicrobial and Blood Repellent Finishes for Cotton and Nonwoven Fabrics Based on Chitosan and Fluoropolymers," Textile Research Journal, vol. 69, no. 2, pp. 104-112, 1999.

[67] H. Shao, W.-D. Meng, and F.-L. Qing, "Synthesis and surface antimicrobial activity of a novel perfluorooctylated quaternary ammonium silane coupling agent," Journal of Fluorine Chemistry, vol. 125, no. 5, pp. 721-724, 2004.

[68] T. Suryaprabha and M. G. Sethuraman, "Fabrication of copperbased superhydrophobic self-cleaning antibacterial coating over cotton fabric," Cellulose, vol. 24, no. 1, pp. 395-407, 2017. 

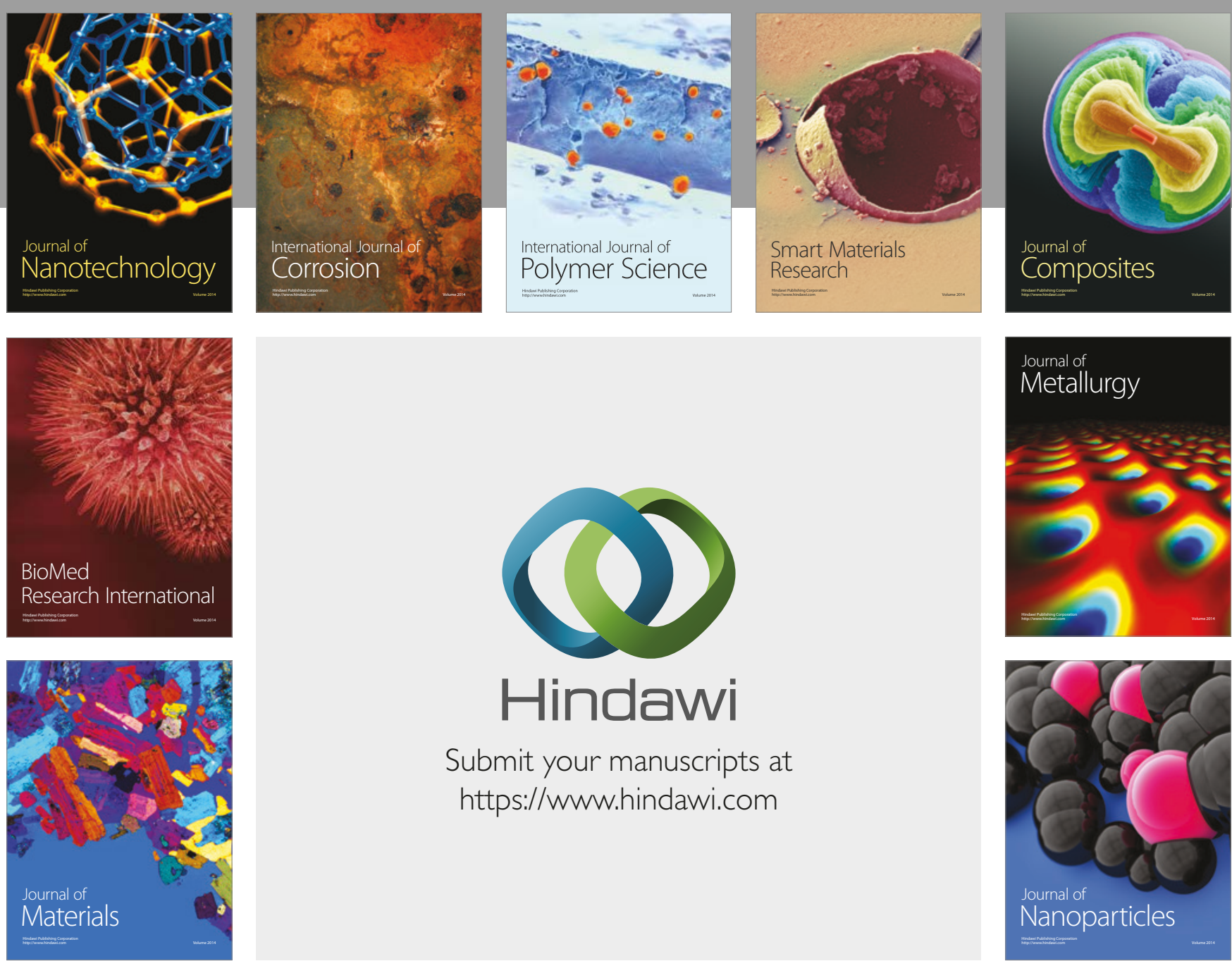

\section{Hindawi}

Submit your manuscripts at

https://www.hindawi.com
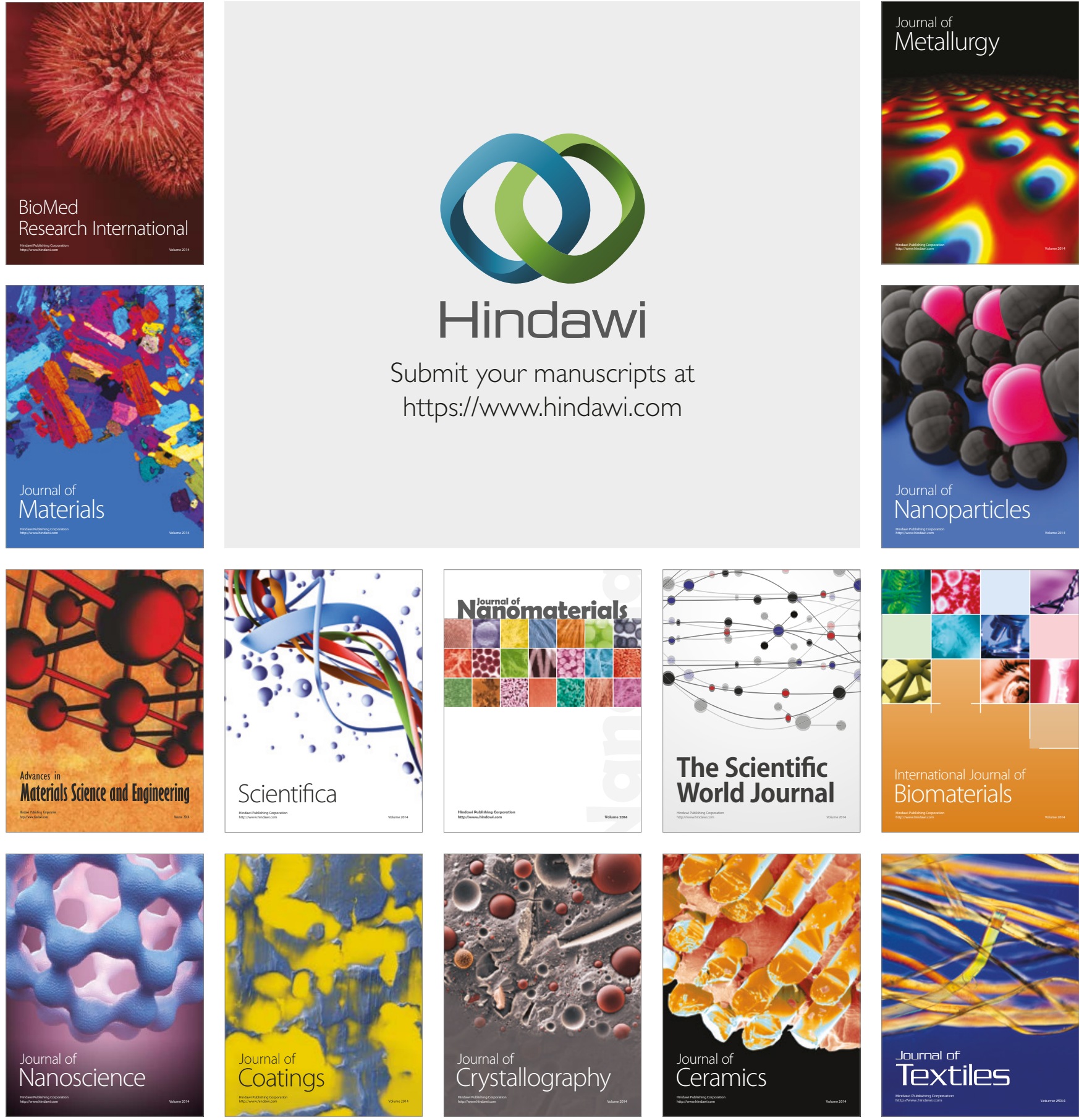

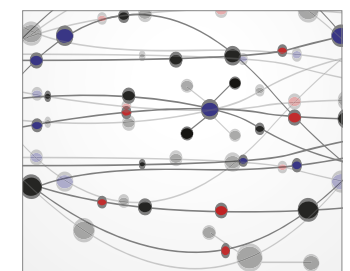

The Scientific World Journal
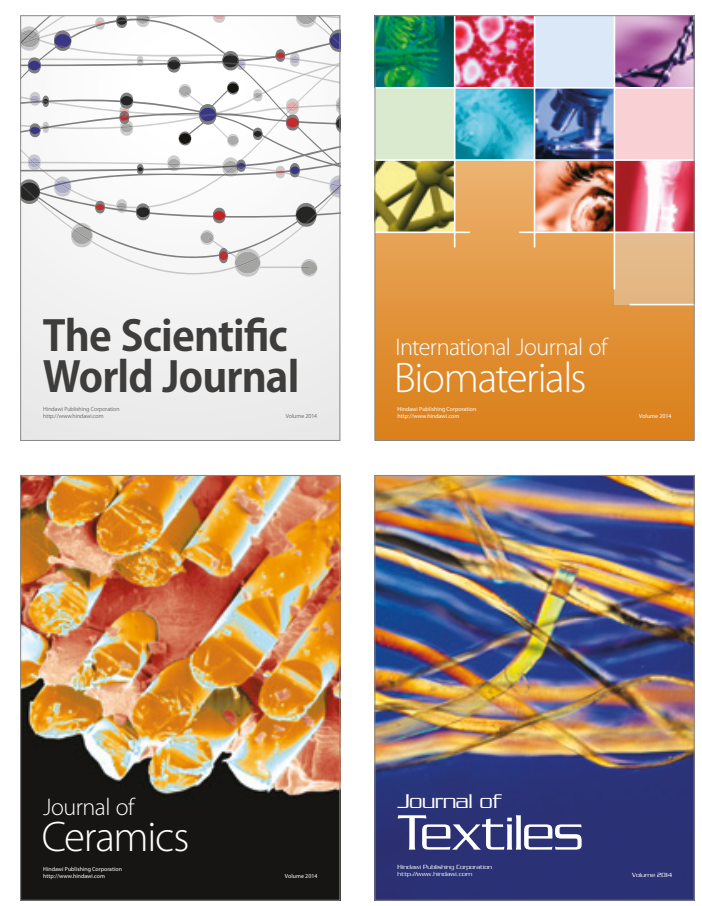